\title{
Mettre en réseau les experts de la qualité
}

\author{
Interview: Fabienne Hohl
}

Vous avez développé un projet novateur en faveur de la qualité qui a fait ses preuves dans la pratique? Le Swiss Quality Award 2016 vous offre une plateforme idéale pour le présenter à un vaste public spécialisé. Nous avons rencontré le Dr Christophe Luthy des Hôpitaux Universitaires de Genève, qui nous parle des répercussions du prix attribué en 2013 au Réseau douleur.*

\section{Votre équipe du Réseau douleur a remporté le Swiss Quality Award en 2013 avec son projet "Agissons ensemble contre la douleur!» qui vise à améliorer la prise en charge de la douleur dans les soins hospita- liers. Comment, d'après vous, ce projet s'est-il distingué?}

Les membres du jury du Swiss Quality Award sont mieux placés que moi pour répondre à cette question! J'espère que l'enthousiasme et la créativité que le Réseau douleur cherche à transmettre aux collaborateurs interdisciplinaires des Hôpitaux Universitaires de Genève (HUG) ont été identifiés comme des éléments essentiels de notre approche de la qualité! Améliorer la prise en charge de la douleur est un processus de longue haleine. Il convient notamment de stimuler les échanges d'idées et le partage des compétences à de multiples niveaux. Il convient de créer des synergies entre les différentes ressources qui existent dans une institution comme les HUG, tant en personnels motivés et formés qu'en moyens techniques. Tout ceci afin que ces ressources soient plus largement utilisées pour soulager les malades.

Qu'est-ce qui vous a poussé à inscrire votre projet au Swiss Quality Award et pas à une autre plateforme? L'audience offerte par cette plateforme pour faire connaître nos actions et établir des liens avec d'autres collègues a constitué un élément déterminant. Nous avons raisonné en termes d'audience locale mais aussi plus largement en termes d'audience en Suisse et à l'étranger.

Qu'est-ce que ce prix a apporté au Réseau douleur? Ces dernières années, nos collaborations interdisciplinaires se sont étendues au sein même des HUG mais également avec des partenaires extérieurs comme avec

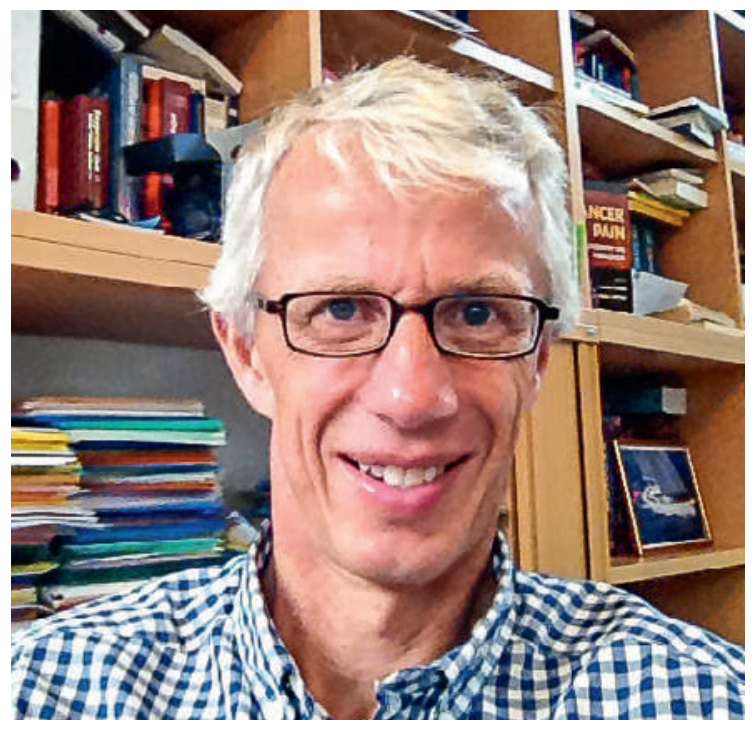

Dr Christophe Luthy, président du Réseau douleur des Hôpitaux Universitaires de Genève: "Le Swiss Quality Award nous a offert une audience locale mais aussi plus largement une audience en Suisse et à l'étranger.»

des écoles professionnelles, avec des organisations de soins à domicile et avec d'autres hôpitaux dans les cantons de Vaud, de Neuchâtel et du Valais notamment. Nous avons également été honorés par un premier prix attribué par la Société Suisse d'Etudes et de traitement de la Douleur (SSED/SGSS).

Qu'y a-t-il de nouveau au sein du Réseau douleur depuis l'attribution du Swiss Quality Award 2013? Le Réseau douleur est en évolution régulière (www. hug-ge.ch/reseau-douleur). Brièvement, nous avons encore renforcé l'utilisation de la douleur comme un indicateur institutionnel de la qualité des prises en charge. Ceci principalement par le biais de formations et d'enquêtes conduites auprès des collaborateurs multi- 
disciplinaires et des malades. Par ailleurs, nous travaillons à diffuser et à développer du matériel d'information et d'aide à la décision. Ces derniers 18 mois, un accent particulier a été porté afin d'améliorer la prise en charge de la douleur induite par les soins (amélioration des pratiques cliniques et de la documentation dans les dossiers des malades) et sur l'antalgie postopératoire (formation des professionnels, coordination interprofessionnelle, diffusion des nouvelles recommandations institutionnelles).

\section{Quelles seraient vos priorités en matière de qualité des soins?}

La nature des soins modernes est en mutation à de multiples niveaux: possibilités de la médecine, évolution des demandes et du profil des malades, interdisciplinarité, coordination entre les hôpitaux et les soins ambulatoires, modes de remboursement, contrôle budgétaire par exemple. Personnellement, j'attache une grande importance à lier les soins à la recherche et réciproquement afin de transférer les connaissances théoriques dans la pratique et réciproquement. Les progrès de la qualité des soins et la maîtrise des coûts imposent cette ouverture et ces collaborations. Pour donner des exemples concrets, les projets qui étudient le partenariat avec le malade et sa satisfaction ainsi que l'optimalisation des modes d'organisation interdisciplinaire des soins constituent des priorités selon moi. Mes deux mots clés sont donc "partenariat avec les malades» et «interdisciplinarité»! Ceci car la médecine d'aujourd'hui nécessite un échange d'informations pour un meilleur

\section{Remportez vous aussi le Swiss Quality Award!}

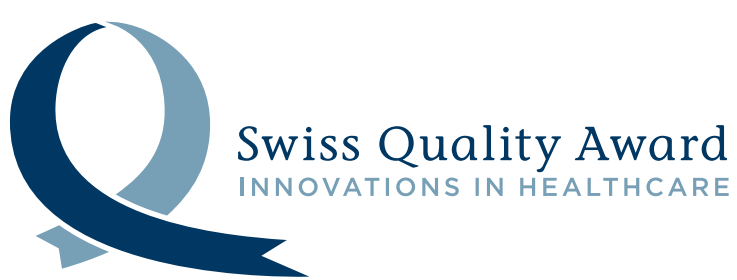

Les idées novatrices en faveur de la qualité dans le domaine de la santé méritent d'être connues - c'est là l'objectif du Swiss Quality Award, qui entend établir un lien entre les pionniers de la qualité, faire connaître des idées ayant fait leurs preuves et nourrir le débat spécialisé. Le Swiss Quality Award récompense les meilleurs projets dans les catégories secteur ambulatoire, secteur hospitalier et projet intersectoriel, dotée chacune d'un prix de 10000 francs. En outre, une récompense d'une valeur de 2000 francs est décernée au meilleur poster. Inscrivez donc sans plus attendre votre projet! Le délai d'inscription est fixé au 8 janvier 2016. Vous trouverez de plus amples informations sur www.swissqualityaward.ch.

consentement et un meilleur partenariat. Les prises en soins complexes nécessitent une attention aux aspects cliniques comme aux aspects organisationnels. La collaboration médicale avec les autres professionnels de santé, mais aussi la collaboration entre les soins ambulatoires et les soins hospitaliers, constituent donc des domaines d'amélioration à fort impact sur la qualité des soins.

\section{Hommage au Prof. Christoph Röder}

Le Dr Christoph Röder nous a quittés subitement le 23 août 2015. Son décès nous a profondément touchés et laisse un grand vide. Christoph Röder était directeur de l'Institut pour la recherche évaluative en médecine de I'Université de Berne qu'il représentait non seulement lors du Swiss Quality Award, mais aussi lors du Symposium national pour la gestion de la qualité dans le secteur de la santé. C'était un homme engagé et enthousiaste aux propositions innovantes. Son humour et sa collégialité ont marqué le secteur de l'assurance qualité en médecine. Grâce à son excellent réseau, il a su initier des collaborations fructueuses et inviter des orateurs passionnants au symposium. Nous lui en sommes très reconnaissants. Christoph, tu vas nous manquer.

Les organismes responsables du Swiss Quality Award: Société Suisse pour le Management de Qualité dans la Santé Institut pour la recherche évaluative en médecine de I'Université de Berne FMH, département Données, démographie et qualité / Académie suisse pour la qualité en médecine 